\title{
Diacronie
}

Studi di Storia Contemporanea

$N^{\circ} 12,4 \mid 2012$

Sulle tracce delle idee

\section{La repressione del movimento contadino in Sicilia (1944-1950)}

\section{Gabriele Montalbano}

\section{(2) OpenEdition}

Journals

\section{Edizione digitale}

URL: http://journals.openedition.org/diacronie/2556

DOI: 10.4000/diacronie. 2556

ISSN: 2038-0925

\section{Editore}

Association culturelle Diacronie

\section{Notizia bibliografica digitale}

Gabriele Montalbano, «La repressione del movimento contadino in Sicilia (1944-1950) », Diacronie [Online], N 12, 4 | 2012, documento 10, online dal 29 décembre 2012, consultato il 10 décembre 2020. URL : http://journals.openedition.org/diacronie/2556 ; DOI : https://doi.org/10.4000/diacronie.2556 


\section{Diacronie}

\section{0/}

\section{La repressione del movimento contadino in Sicilia (1944-1950)}

Gabriele MONTALBANO*

Il paesaggio agrario della Sicilia nel secondo dopoguerra era ancora dominato dalle grandi proprietà terriere. Il movimento contadino, incentivato dal decreto Gullo del 19 ottobre 1944 che distribuiva $i$ terreni incolti o mal coltivati a cooperative di contadini, si oppose a questo sistema attraverso l'occupazione dei latifondi. Gli agrari si difesero utilizzando vie legali, ma non solo. L'articolo descrive il patto agrariomafioso messo in atto per reprimere questa mobilitazione. Vengono analizzate in particolare le uccisioni dei dirigenti del movimento contadino Placido Rizzotto e Giuseppe Maniaci, per mostrare il grado di collaborazione che si venne ad instaurare tra alcuni esponenti delle forze di pubblica sicurezza e la mafia.

\section{La repressione giudiziaria}

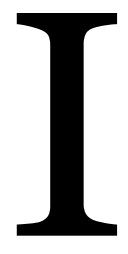

1 movimento contadino siciliano del secondo dopoguerra fu un grande fattore di cambiamento per la realtà agraria isolana, allora dominata dai latifondisti e dai gabelloti che ne gestivano i feudi. La fine del latifondo e dei rapporti di potere su di esso basati furono il principale obiettivo delle lotte contadine ma i grandi proprietari terrieri dell'interno siciliano si opposero in ogni maniera alle rivendicazioni bracciantili, benché quest'ultime fossero in parte incoraggiate dal decreto Gullo n. 279 del 19 ottobre 1944, che limitava la proprietà privata in relazione alla produzione agricola. Affinché si applicassero correttamente e in tempi brevi le 
disposizioni previste dal decreto che dava in concessione le terre ${ }^{1}$, i contadini occuparono simbolicamente i terreni incolti dei latifondi. Per gli agrari difendere il latifondo dalle occupazioni contadine significava difendere il proprio peso politico e ruolo sociale. La guerra legale che si sviluppò contro il movimento fu tale che nel 1948 nacque a livello nazionale il centro di assistenza legale per i contadini sotto accusa. Il Comitato di solidarietà democratica ${ }^{2}$ riunì vari avvocati che difesero e assistettero legalmente i lavoratori per le lotte sindacali e i contadini «per la conquista delle terre incolte o mal coltivate per la riforma agraria»3. Una delle battaglie legali più importanti sostenute dal Comitato fu quella per «far riacquistare il diritto di voto [...] a coloro che l'avevano perduto in seguito a condanne per motivi politici» 4 , come quelle che venivano comminate ai dirigenti o ai membri più attivi delle organizzazioni che rivendicavano il diritto alla terra occupando i latifondi. Le occupazioni fino al 1949 non avevano l'intento di prendere possesso effettivo del terreno ma servivano per mettere pressione alle commissioni provinciali, che dovevano valutare le domande per la concessione dei lotti. I contadini andavano in massa dal paese fino ai feudi incolti e lì piantavano una bandiera che poteva essere il tricolore italiano, quella rossa comunista o bianca dei democristiani. La manifestazione poteva prevedere atti simbolici come tracciare un solco con l'aratro, il dissodamento di una piccola parte del terreno occupato e, soprattutto dopo il 1948, altri lavori agricoli che rendevano l'occupazione un vero e proprio sciopero alla rovescia dove i contadini lavoravano gratuitamente nei terreni incolti per sottolineare la necessità di lavoro e di terra alle autorità competenti. Nonostante la manifestazione fosse pacifica e durasse qualche ora, al ritorno dai campi o direttamente durante lo svolgimento dell'occupazione, le forze dell'ordine fermavano i partecipanti. Nel 1949 e nel 1950 le occupazioni da simboliche divennero effettive e i contadini iniziarono a seminare grano in quei terreni che ancora non venivano conferiti alle cooperative richiedenti.

\footnotetext{
${ }^{1}$ «Le associazioni di contadini regolarmente costituite in cooperative o in altri enti, possono ottenere la concessione di terreni di proprietà privati o di enti pubblici che risultino coltivati $o$ insufficientemente coltivati in relazione alle loro qualità, alle condizioni agricole del luogo e alle esigenze culturali [sic] dell'azienda in relazione con le necessità della produzione agricola nazionale» art. 1 del decreto legislativo luogotenenziale 19 ottobre 1944, n.279 (in Gazzetta ufficiale, serie speciale 4 novembre 1944 n.77).

2 Il Comitato provinciale di Palermo fu il primo in Sicilia e dopo la formazione degli altri comitati nelle varie città siciliane, nel 1951 divenne regionale con funzione di coordinamento tra gli altri.

3 SGRÒ, Vincenza, Il Comitato di solidarietà democratica di Palermo in "Bollettino dell'Istituto Gramsci Siciliano", Palermo, 1999, p. 76.

4 Ibidem, p. 74.
} 


\subsection{Il caso di Campofiorito}

Le motivazioni che spinsero all'occupazione dei latifondi si possono leggere nei verbali delle deposizioni di alcuni contadini di Campofiorito (paese agricolo all'interno della provincia palermitana, non lontano da Corleone) interrogati per reato di invasione di terreni avvenuta il 15 novembre 1949. Scrivono i carabinieri:

i soci della Cooperativa Lavoro Proletario di Campofiorito più volte riuniti decidevano di occupare i terreni incolti o memi [sic] incolti, perché, pur avendo più volte richieste ai competenti organi, l'assegnazione delle terre alla predetta Cooperativa, non erano mai stati accontentati [...] hanno dichiarato concordemente di essersi portati alla località Giardinello procedendo alla occupazione reale di quella zona essendo tutti senza lavoro e disoccupati5.

Il processo celebratosi per questa occupazione avvenuta nel territorio di Corleone ha dei tratti che lo rendono abbastanza esemplare per comprendere l'atteggiamento che ebbero le varie parti in causa nelle battaglie legali tra proprietari e contadini. Vi è una netta differenza tra la versione depositata dai contadini interrogati, dai testimoni oculari dell'occupazione e quella dei proprietari. La scelta dell'occupazione del latifondo nasce dal plurimo rifiuto della commissione competente che blocca sul nascere le possibilità di lavoro per i braccianti, benché sia accertato dai carabinieri che le terre oggetto di occupazione fossero incolte e abbandonate dal proprietario. Quest'ultimo nel denunciare i contadini per invasione aggravata asserì che i suoi terreni ricevettero un danno ingente causato oltre che dal «dissodamento di terreni che non potevano essere dissodati, sia per ragioni culturali [sic], sia per motivi di teca [terra?] agraria, è stato anche costituito dal calpesto di grande numero di persone e rispettivi animali, nonché del pascolo di questi ultimi» ${ }^{6}$. Alla dichiarazione del proprietario - che non era fisicamente presente all'occupazione - dei danni subiti e da risarcire faceva da contradditorio la testimonianza degli stessi militari che quel giorno sorvegliarono l'occupazione. Dichiararono: "gli occupanti furono seguiti dai militari di questa stazione per accertarne luogo e sviluppo [...]. Non furono commesse devastazioni o danneggiamenti di sorta» ${ }^{7}$. Il carattere pacifico dell'occupazione è ribadito anche da altri 2 testimoni, un padre e un figlio, che lavoravano in quel feudo e diedero una

5 ARCHIVIO GRAMSCI SICILIANO (d'ora in poi AGS), fondo Comitato Solidarietà Democratica (CSD), b. 11, fasc. 11, Procedimento penale contro Alfano Giuseppe fu Francesco ed altri 41 imputati di invasione aggravata di terreni. Udienza del 13/12/1951, pp. 3-4, 8.

${ }^{6}$ Ibidem, pp. 13-14.

7 Ibidem, p. 6. 
sommaria descrizione degli avvenimenti. Il padre dichiarò che erano lì «quando ne fu eseguita l'invasione ad opera di oltre 250 persone, venute apposta da Campofiorito, con la bandiera in testa. Io lavoravo con mio figlio in prossimità delle case, dove non fui molestato da alcuno, e non ho conosciuto nessuno degli invasori, tra i quali vi erano molte donne [...]»8. Era un movimento organizzato e pacifico, consapevole delle ragioni che lo muovevano e con un obiettivo chiaro e preciso: avere la possibilità di lavorare le terre incolte. L'uso della bandiera, di qualunque tipo, era un elemento comune e costante durante le occupazioni e dava a quell'azione collettiva un riferimento alla nazione o al partito di riferimento. In un contesto come quello delle campagne siciliane in cui la soggezione paternalistica era la base dei rapporti tra contadino e proprietario, l'uso di una bandiera per un atto simile era un tentativo di spersonalizzazione del conflitto che eliminava ogni tipo di inibizione data dal legame personalistico con il proprietario da contestare 9 . Era un salto di qualità che permise ai contadini di uscire da una soggezione imposta dall'autorità degli agrari per potersi riallacciare a movimenti di respiro regionale e nazionale che andassero oltre le particolarità e difendessero il loro diritto alla terra. La disgregazione del blocco agrario era probabilmente irreversibile nella lenta avanzata della società di massa nelle campagne meridionali, l’inasprimento dell'atteggiamento degli agrari è dato quindi dalla consapevolezza di essere in una posizione di difesa senza reali prospettive.

Nonostante le varie testimonianze delle forze di polizia come dei testimoni civili che confermano il non danneggiamento delle terre occupate, il proprietario di quest'ultime dichiarò: «il danno arrecato al fondo è di circa lire trentamila. Chiedo la punizione dei colpevoli ${ }^{10}$. L'esplicita richiesta di punizione dei colpevoli lascia intendere una volontà di rivalsa verso l'insubordinazione contadina. A fronte di queste dichiarazioni e testimonianze il Pubblico Ministero rinviò a giudizio ${ }^{11}$ gli imputati per invasione aggravata. Nell'argomentare la sua decisione il PM spiegò:

la circostanza che trattasi di terre incolte ed abbandonate a pascolo, anche se sussistente, non influisce sulla configurazione attribuita al fatto. [...] Irrilevante ̀̀

\footnotetext{
8 Ibidem, p. 25. Similmente dichiarò il figlio: «non fu loro opposta alcuna resistenza, anche perché, per la verità, nessuna molestia essi diedero a me ed a mio padre, che continuammo a lavorare pei fatti nostri. [...] Nessun danno fu arrecato alle terre». Ibidem, p. 28.

${ }^{9}$ SANTINO Umberto, Storia del movimento antimafia. Dalla lotta di classe all'impegno civile, Roma, Editori Riuniti, 2000, passim.

10 AGS, fondo CSD, b. 11, fasc. 11 Procedimento penale contro Alfano Giuseppe [...] p. 30 (corsivo dell'autore).

${ }^{11}$ Il Pubblico Ministero poteva rinviare a giudizio in quanto era ancora in vigore il codice di procedura penale Rocco del 1930. Prima di questa data il PM aveva potere inquisitorio e poteva decidere il rinvio a giudizio o l'assoluzione.
} 
che la permanenza degli invasori nel fondo si sia protratta solo per poche ore e che l'attività degli imputati si sia limitata al dissodamento [...] ${ }^{12}$.

Il fatto che le terre occupate dai contadini fossero incolte non viene considerato come un elemento da tenere presente in ambito giudiziario, quando in realtà era centrale per comprendere le motivazioni dell'occupazione da parte di braccianti disoccupati. In questo caso l'occupazione si svolse in maniera ordinata e benché si fossero generate delle ripercussioni legali contro i contadini, non si può parlare di uno scontro diretto con le forze dell'ordine, che anzi si assicurarono del corretto svolgimento dell'occupazione. Ma non sempre le occupazioni dei latifondi si svolgevano così serenamente.

\section{La repressione poliziesca}

Le deposizioni di cui ci avvarremo adesso riguardano il processo contro Pio La Torre $^{13}$ e altri contadini a causa di un'occupazione che vide le forze dell'ordine assumere un atteggiamento ben più violento di quello precedentemente analizzato nel caso degli occupanti di Campofiorito. Questa occupazione fu molto più grande e importante di quella precedentemente descritta; il 10 marzo 1950 Pio La Torre, alla testa di un corteo di circa seimila persone, decise l'occupazione di quasi duemila ettari del feudo Santa Maria del Bosco nei pressi di Bisacquino, paese dell'interno siciliano. Alla sera, al rientro, i contadini occupanti vennero fermati dalle forze di polizia inviate dal prefetto di Palermo, Angelo Vicari, e la forza pubblica aprì il fuoco. La tensione era già molto alta in quanto la manifestazione del 10 marzo aveva l'aria di un atto di sfida nei confronti del prefetto Vicari che voleva scorporare i tremila ettari di terre incolte già seminate dai contadini durante la stagione precedente, riconsegnare i terreni e il loro prodotto ai proprietari e far decidere a questi ultimi che terreni dare in concessione ai contadini. Questa manovra filo-padronale trovò la dura opposizione di tutto il movimento contadino. Dalle testimonianze dei lavoratori della terra è possibile osservare la ricerca dello scontro da parte delle forze dell'ordine comandate dal commissario Panico e l'uso violento che fecero della forza pubblica. Le deposizioni, che

12 Ibidem, p.37. (corsivo dell'autore)

13 Importante dirigente del movimento contadino e del Partito Comunista, divenne nel 1947 funzionario della Federterra. Su Pio La Torre si vedano: MANCUSO, Davide, «Biografia di Pio La Torre», URL: <http://www.piolatorre.it/Pio_La_Torre_biografia.asp> [consultato il 17 novembre 2012]; BASCIETTO, Giuseppe, CAMARCA, Claudio, Pio La Torre una storia italiana. La vita del politico e dell'uomo che sfidò la mafia, Reggio Emilia, Aliberti, 2008; RENDA, Francesco, Portella della Ginestra e la guerra fredda. I cento anni della CGIL siciliana, conversazioni con Antonio Riolo, Roma, Ediesse, 2007. 
si svolsero nell'antico carcere borbonico dell'Ucciardone di Palermo rivelano la tragicità di quell'evento. Un contadino, Gioacchino D’Accursio, che vide da vicino il sorgere del conflitto a fuoco lasciò questa importante dichiarazione:

[...] Udii per prima gridare le donne, e poi il clamore si fece generale. [...] I militari sparavano contro la folla. Io vidi alcuni di questi puntare le armi contro la folla e sparare a ventaglio [...] Noi avevamo solo arnesi da lavoro [...]14.

La gravità di quell'episodio denota come all'interno delle forze di polizia vi fosse una chiara avversione contro la mobilitazione contadina, fino al punto di sparare ad una folla di donne e uomini disarmati. La dichiarazione di D'Accursio se ci dà in poche righe l'idea di come si svolse quella sparatoria, non dice come iniziò, quale fu la causa scatenante che spinse dei poliziotti a non trovare altra soluzione mediatrice che usare le armi contro i civili. Questo testimone era abbastanza vicino al centro dell'azione per sentire le prime grida ma non da poterne vedere la causa. La testimonianza dell'assessore comunista di Bisacquino, Nicola Sicula, presente all'occupazione chiarisce l'accaduto:

[...] il La Torre andò incontro al tenente, seguito da me, e disse subito che il corteo stava per sciogliersi [...] il Commissario disse alle donne di abbassare le bandiere. Una donna rispose - questo mai ! - [...]. Ne seguì una colluttazione [...] vidi i carabinieri che erano sui camion precipitare a terra sparando ed avanzando per caricare la folla $[\ldots]^{15}$.

\subsection{Lotte di emancipazione}

In questo coinvolgimento delle classi contadine all'attivismo sindacale e politico risalta il ruolo delle donne all'interno del movimento contadino per le terre occupate. Come si evince dalla precedente dichiarazione erano le donne ad essere in prima fila con le bandiere e le prime ad opporsi alle prepotenze dei carabinieri. Sempre presenti alle occupazioni dei latifondi con i bambini accanto, sottolineavano indirettamente con la loro presenza il carattere non violento della manifestazione e ricoprivano una funzione di mediazione tra $\mathrm{i}$ contadini più turbolenti e i poliziotti. A riprova della forte spinta delle lotte contadine per la democratizzazione della società isolana del dopoguerra, questa esperienza risulterà molto importante per l'emancipazione

14 Ibidem, b. 36, fasc. 1, p.5 (corsivo dell'autore).

15 Ibidem, p. 156 (corsivo dell'autore). 
femminile in Sicilia. Qualche anno dopo, nel 1953, si terrà a Palermo il primo congresso regionale delle donne siciliane, «con oltre 1.500 delegate elette dalle donne di tutta la Sicilia»16. La carica di modernità, democrazia e giustizia sociale presente nel movimento contadino siciliano lo rende a tutti gli effetti, come dimostrato dal sociologo e storico Umberto Santino ${ }^{17}$, uno dei più significativi movimenti collettivi dell'antimafia. Nonostante la lotta del movimento per l'applicazione di leggi dello Stato venne pesantemente condannato dai processi che si intentarono per le occupazioni dei latifondi. I dati che si possono reperire dalle carte del Comitato Regionale di Solidarietà Democratica indicano che, considerando complessivamente le lotte per la riforma agraria, «i contadini denunziati furono 3.185, quelli assolti 386, quelli processati 2.323, e condannati complessivamente a 293 anni e 36 mesi di reclusione e 7.543.280 lire di multe» ${ }^{18}$.

\section{La formazione del blocco agrario-mafioso}

Prima di poter descrivere le modalità e le motivazioni del terrorismo agrariomafioso bisogna spiegare come si è formato tale blocco di potere ${ }^{19}$. Il contesto del dopoguerra sicuramente aiuta a comprendere la situazione di forte instabilità che imperversava nelle campagne siciliane. L'emergere del movimento contadino per la terra, prima in maniera turbolenta e indisciplinata poi con forma sempre più organizzata e strutturata attraverso la cooperazione agricola, allarmò tutti i grandi feudatari che temettero sempre di più l'avanzata contadina e il rischio che questa potesse spazzare via il loro potere economico e sociale. Al contempo il potere dei mafiosi trovò nella situazione d'instabilità un'occasione ottima per ristrutturarsi, anche

${ }^{16}$ LO MONACO, Vito, «50 anni dalle lotte per la riforma agraria; l'occupazione del Feudo S. Maria del Bosco; i fatti di Bisacquino»,

URL: < http://www.piolatorre.it/quaderni/quadernoo4.asp > [consultato il 17 novembre 2012] ${ }^{17}$ SANTINO Umberto, Storia del movimento antimafia. Dalla lotta di classe all'impegno civile, Roma, Editori Riuniti, 2000.

${ }^{18}$ SGRÒ, Vincenza, Il Comitato di solidarietà democratica di Palermo, cit., p. 79. «Dal 1949 al 1955 nelle lotte in difesa delle libertà civili nella sola provincia di Palermo vi furono 884 lavoratori arrestati o fermati, 5.065 denunziati all'autorità giudiziaria, 1.886 condannati per 681 anni di carcere. Negli stessi anni in tutta la Sicilia vi furono 2.916 lavoratori arrestati o fermati, 7.708 denunziati, 4.960 condannati, per 1.330 anni di carcere».

${ }_{19} \mathrm{Si}$ veda in merito: SANTINO, Umberto, Storia del movimento antimafia ,op. cit.; RENDA, Francesco, Il movimento contadino in Sicilia e la fine del blocco agrario nel Mezzogiorno, Bari, De Donato, 1976; ID., Movimenti di massa e democrazia, Bari, De Donato, 1979; ID., Storia della Sicilia dal 1860 al 1970. Palermo, Sellerio, 1984; MARINO, Giuseppe Carlo, Storia del separatismo siciliano, Roma, Editori Riuniti, 1979. 
grazie al ruolo che ebbero i capimafia all'indomani dello sbarco alleato in Sicilia ${ }^{20}$, a cui venne dato il compito di riportare l'ordine sociale in una situazione fortemente critica. Furono in effetti diverse le nomine di mafiosi a capo di comuni da parte dell'amministrazione militare americana ${ }^{21}$. Il potere della mafia è visto quindi come garante e stabilizzatore delle tensioni sociali che sconvolgono l'Isola nel dopoguerra, «ai capimafia la prima legittimazione venne dal loro insediamento operato da Charles Poletti nell'amministrazione dei vari comuni»22. Contro il banditismo - endemico nelle campagne - la mafia era utile sia all'amministrazione alleata, sia agli agrari per mantenere una forza di controllo che potesse debellare la minaccia dei banditi ${ }^{23}$ e dei contadini che rivendicavano la terra.

Questa necessità di ordine, controllo e contenimento spinse le autorità alleate, e poi gli agrari, ad allearsi con la mafia: «nella saldatura tra mafia e potere trovarono una spiegazione la nascita e la liquidazione del banditismo»24. Il movimento contadino fu visto come una fonte di instabilità da abbattere in quanto, oltre a minare le basi del potere feudatario, metteva in pericolo anche l'autorità stessa della mafia. La funzione intermediaria del gabelloto mafioso, o del caporalato sui braccianti, garantiva un forte assoggettamento da parte delle classi deboli contadine. Le rivendicazioni portate avanti dal movimento nel tentativo di cambiare la gestione dei terreni e di modificare i rapporti di forza tradizionali delle campagne intaccava direttamente il potere dei mafiosi. Molti di essi in quegli anni diventarono gestori dei feudi dei grandi proprietari, sancendo così l'alleanza di fatto tra agrari e mafiosi. Si ebbe così la formazione di un vero e proprio blocco di potere agrario-mafioso, «un fronte comune per fronteggiare il nemico di sempre: il movimento dei contadini»25. Soprattutto nella Sicilia occidentale, la Sicilia del feudo, si nota un vero e proprio arruolamento da parte degli agrari dei mafiosi, che diventano ora i campieri di fiducia dei grandi proprietari. Esemplare è la

\footnotetext{
20 «la mafia non è stata come spesso si scrive, un regalo degli Alleati[...] è risorta in Sicilia non per colpa esterna ma perché agrari, separatismo [...] aprirono le porte della tolleranza o della collaborazione». RENDA, Francesco, Portella della Ginestra e la guerra fredda. I cento anni della CGIL siciliana, conversazioni con Antonio Riolo, Roma, Ediesse, 2007, p. 148.

${ }^{21}$ Durante i sette mesi di governo alleato in Sicilia, si ebbe una repentina scalata al potere dei mafiosi nelle amministrazioni comunali. «Siamo di fronte ad un'assunzione diretta del potere da parte della mafia, una vera e propria criminocrazia formale, e questo non era mai avvenuto prima , in modo così eclatante». SANTINO, Umberto, Storia del movimento antimafia, cit., p. 134 .

${ }_{22}$ CASARRUBEA, Giuseppe, Storia segreta della Sicilia, dallo sbarco alleato a Portella della Ginestra, Milano, Bompiani, 2007, p. 41.

23 «Con le diverse decine di bande armate che infestavano l'isola da secoli furono regolati i conti. Passarono sotto l'appalto mafioso e i padrini le eliminarono tutte, una dopo l'altra, con accanita sistematicità». Ibidem, p. 39.

24 Ibidem, p. 89.

${ }^{25}$ SANTINO, Umberto, Storia del movimento antimafia, cit., p. 154.
} 
nomina di Calogero Vizzini, il più potente capomafia siciliano dell'epoca, come gestore del feudo Micciché della principessa di Trabia Giulia Florio D’Ontes che, essendo in parte incolto e abbandonato, era richiesto dai contadini poveri del paese riuniti nella cooperativa "Libertà". Vizzini riprendeva possesso di quel terreno che era già stato sotto la sua gestione anni prima e che aveva dovuto abbondare durante il fascismo a causa della repressione di Mori. Tutti i capimafia presero il controllo dei feudi più importanti: i principi Lanza di Trabia affidarono il loro feudo a Giuseppe Genco Russo che era appena uscito di galera; Vanni Sacco capomafia di Camporeale, ebbe in gestione il feudo Parrino; anche Luciano Leggio divenne gabelloto di un feudo dalle parti di Corleone, benché avesse già «un mandato di cattura per essere stato accusato di gravissimi reati» ${ }^{26}$. Quasi ogni feudo aveva la sua protezione mafiosa di riferimento.

\section{1 «Come una guerra» 27}

Questo pactum sceleris che formava il blocco agrario-mafioso passò ben presto ad azioni omicide contro i dirigenti del movimento contadino. Le sedi dei partiti di sinistra furono il primo bersaglio che si decise di colpire per dare un messaggio chiaro alla popolazione e ai contadini iscritti; il più delle volte le sedi del PCI e quelle del PSI coincidevano per ragioni di economia e gestione soprattutto nei paesi poveri dell'interno siciliano ${ }^{28}$. I dirigenti socialisti furono quelli maggiormente vittime delle violenze mafiose, in quanto si cercava non solo di intimidirli nella loro lotta contro la mafia e il feudo, ma anche di «indurli a rompere il patto con i comunisti» ${ }^{29}$, volendo così spezzare l'alleanza social-comunista che era una minaccia reale al potere mafioso. I giornali locali dell'epoca, come «La Voce della Sicilia» e «L'Ora» riportavano quasi quotidianamente notizie di attentati, intimidazioni, uccisioni, sparatorie contro i dirigenti del movimento contadino, dei partiti di sinistra e quelli sindacali. La strategia terroristica mafiosa era volta a smantellare le organizzazioni contadine a qualunque costo. L'attentato che può essere considerato come una dichiarazione di guerra della mafia ai partiti social-comunisti, fu quello di Villalba del 16 settembre 1944. L'occasione fu un comizio del partito comunista proprio nel paese del capomafia Vizzini in cui a parlare era Girolamo Li Causi, il segretario del partito in Sicilia. Durante il comizio, Li Causi denunziò il patto tra agrari e mafios,i prendendo ad esempio proprio il citato feudo Micciché, gestito da Vizzini. Quest'ultimo rispose immediatamente alla

\footnotetext{
${ }^{26}$ Ibidem, p. 155 .

${ }_{27}$ Titolo di uno speciale del giornale «L'Ora» del 27 aprile 1967 sulle lotte contadine.

${ }^{28}$ SANTINO, Umberto, Storia del movimento antimafia, cit., passim.

29 Ibidem, p. 171.
} 
provocazione con una sparatoria nella piazza del comizio a cui prese parte anche il sindaco democristiano di Villalba, Beniamino Farina nipote di Vizzini, che lanciò una bomba a mano contro i comunisti: «Li Causi si reca nella tana del lupo e don Calò Vizzini risponde alla sfida con i suoi mezzi e il suo linguaggio»30. Il caso dell'attentato di Villalba preannunciava il pesante intreccio tra la mafia e alcuni esponenti della Democrazia Cristiana che divenne ben presto una costante della politica siciliana. I comunisti attraverso i loro giornali e le dichiarazioni pubbliche accusarono - in merito all'attento a Li Causi - la connivenza democristiana con i poteri mafiosi. Bernardo Mattarella, dirigente della DC, contestò le accuse dei comunisti sul giornale «Il Popolo» del 24 settembre difendendo il suo partito e sminuendo il carattere politico dell'attentato mafioso. Per Mattarella il conflitto a fuoco era causato da faide tra famiglie rivali e non da motivazioni politiche per reprimere i comunisti. Non dello stesso avviso era il partito socialista che dichiarò:

La provincia di Caltanissetta è da un anno praticamente nelle mani dei democristiani. Perché le cricche reazionarie, latifondiste, fasciste e maffiose [sic] hanno potuto continuare a dominare indisturbate? [...] È la tradizione: la mafia siciliana può vivere al patto d'essere favorita dalle Autorità. Il partito democristiano si prepara ad ereditare questa funzione, che fu in altri tempi dei partiti sedicenti democratici liberali ${ }^{1}$.

Anche le forze di polizia cercarono un accordo con il potere mafioso, rappresentato da don Calogero Vizzini. In documento dell'Office of Strategic Service ${ }^{2}$ si può leggere il grado di connivenza e reciproca protezione che si venne ad instaurare tra di loro:

la Pubblica Sicurezza e i Carabinieri sono apertamente favorevoli all'improvviso interesse dell'alta mafia per la situazione della legge dell'ordine e volutamente evitano di investigare sugli omicidi dei suddetti fuorilegge 33.

I dirigenti politici e sindacali che vennero uccisi dalla mafia a causa del loro ruolo nelle lotte contadine furono, secondo le stime della Regione Siciliana che li riconobbe

\footnotetext{
${ }^{30}$ Ibidem, p. 142.

${ }^{31}$ In «La Voce Socialista» del 7 ottobre 1944 (corsivo dell'autore).

${ }^{22}$ L'Office of Strategic Service era l'agenzia di intelligence segreta degli Stati Uniti durante la seconda guerra mondiale, nel 1947 cambiò nome divenendo la CIA (Central Intelligence Agency) che mantiene ancora.

33 OSS, «L'alta mafia combatte il crimine» del 5 aprile 1945, riportato in CASARRUBEA, Giuseppe, Storia segreta della Sicilia, cit., p. 89 (corsivo dell'autore).
} 
come vittime di mafia, 52 in un arco di tempo che va dal 1944 al $1960^{34}$. Senza considerare gli attentati alle sedi, ai raccolti agricoli, ai comizi; senza contare i feriti e coloro che furono ammazzati pur non avendo un ruolo dirigenziale o uccisi per sbaglio a causa delle sparatorie, o non riconosciuti come vittime di mafia, le cifre della violenza mafiosa fanno comprendere che la condizione della Sicilia di quegli anni era non lontana a quella di una zona in stato di guerra civile. Per avere un'idea della violenza complessiva dell'ambiente siciliano del dopoguerra, ai morti ammazzati riconosciuti ufficialmente come vittime di mafia bisogna aggiungere coloro che furono uccisi dalle forze dell'ordine durante le manifestazioni o le contestazioni più cruente, come per esempio quella avvenuta a Caccamo, in provincia di Palermo, dove il 5 agosto del 1946 vi fu uno scontro particolarmente violento per l'ammasso del grano, che vide 4 morti e 21 feriti tra le forze dell'ordine e 20 morti e 60 feriti fra $\mathrm{i}$ contadini35. Inoltre la banda di Salvatore Giuliano, manovrata dalla mafia e dall'eversione neofascista ${ }^{36}$, continuerà a compiere i suoi atti di criminalità, attentati, rapine, sequestri, fino al 1950, anno in cui venne ucciso il bandito Giuliano, in circostanze ancora non del tutto chiarite.

\section{Tra processi e depistaggi}

In questi conflitti tra contadini e agrari, che possono essere veramente considerati come una guerra civile, abbiamo avuto modo di vedere il ruolo repressivo delle forze dell'ordine e della mafia. Altro aspetto molto importante è lo svolgimento dei numerosi processi che si fecero contro gli assassini dei dirigenti del movimento e la relativa scarsità di condanne; molte di quelle uccisioni rimasero infatti impunite. Come fa notare lo storico, il ruolo di copertura di alcuni esponenti dello Stato agli attacchi mafiosi è un’ipotesi già analizzata da Casarrubea ${ }^{37}$. Il depistaggio e lo sviamento delle indagini sembrano essere delle costanti in tutti i processi riguardanti l'uccisione dei dirigenti per mano dei sicari mafiosi. Anche quando vi era il sospetto generale che i

34 Dati presi da FIGURELLI, Michele, PANTANO, Linda, SGRÒ, Enza, Placido Rizzotto e altri caduti per la libertà contro la mafia, introduzione di Salvo Riela agli atti processuali da lui donati all'Archivio dell'Istituto Gramsci Siciliano, Palermo, Istituto Gramsci Siciliano, 2012, pp. 106-108.

35 SANTINO, Umberto, Storia del movimento antimafia, cit., p. 156

${ }^{36}$ La tesi proposta da Casarrubea dimostra il legame con vari esponenti dell'OSS e dei gruppi neofascisti con il banditismo siciliano e la mafia in vista del contenimento e della repressione dei movimenti di sinistra. Il personaggio che garantì l'accordo tra questi due poteri fu l'ispettore capo delle forze dell'ordine in Sicilia Ettore Messana, «di fatto un anello nevralgico del blocco reazionario in Sicilia». in CASARRUBEA, Giuseppe, Storia segreta della Sicilia, cit., p. 96.

37 Il ruolo di Ettore Messana sembra essere centrale in questo accordo tra polizia e mafia, «che servì a garantire l'impunità negli attacchi neofascisti e mafiosi contro la sinistra» in Ibidem. 
mandati degli omicidi potessero essere i feudatari contro cui si muoveva l'azione di quei dirigenti, quasi mai vennero indagati o si indagò vagliando quelle ragioni. Si tentò sistematicamente di sviare le indagini dal movente politico ad uno passionale o di vendetta privata. Le indagini di polizia e carabinieri si svolsero in un'ottica di depoliticizzazione della figura del dirigente ucciso cercando quindi il movente nel passato privato della persona: gelosie, inimicizie, odi di paese furono le piste preferite da indagare. L'ammissione della natura politica dell'omicidio avrebbe significato la rottura di un equilibrio di poteri nella zona ed una scomoda presa di posizione contraria ai poteri forti locali ${ }^{3}$. Si cercava piuttosto di screditare l'ucciso mostrando, quando possibile, problemi giudiziari trascorsi, anche minimi, a cui appigliarsi per ventilare le possibili motivazioni di una vendetta personale.

\subsection{Giuseppe Maniaci}

Prenderemo in esame il caso di Giuseppe Maniaci, esemplare della routine giudiziaria di quei processi, ma che presenta al contempo delle particolarità che permettono di gettare una luce sulla mentalità delle forze dell'ordine rispetto alla mafia locale. Maniaci, iscritto al partito comunista e segretario della Federterra locale, venne ucciso a Terrasini, in provincia di Palermo, vicino a Cinisi, noto paese di mafia, il 22 ottobre 1947. Nel messaggio di segnalazione inviato il giorno dopo alla Procura della Repubblica di Palermo si legge:

ore 13 circa 22 andante ignoti uccidevano colpi di mitra [...] Maniaci Giuseppe fu Salvatore anni 38 del luogo, contadino, segretario Federterra predetto comune. Escludesi movente politico. Si ritiene probabile uccisione per vendetta essendo Maniaci fortemente pregiudicato delitti contro il patrimonio39.

Nella prima comunicazione che le forze dell'ordine locali fanno alla Procura di Palermo è volta all'esclusione del movente politico, senza per altro addurne spiegazioni o chiarimenti di sorta, quasi come per scongiurare immediatamente l’ipotesi più pericolosa da sostenere. La seconda mossa che i carabinieri del luogo fanno è presentare la vittima in una condizione di precedente colpevolezza. Mostrando che il Maniaci fu anch'egli un criminale, si vuole intravedere nell'uccisione un regolamento di

\footnotetext{
$3^{8}$ «La natura politica del delitto interrompeva l'abituale tran tran investigativo, imponeva ritmi nuovi, metteva in dubbio equilibri raggiunti nel territorio [...]» in FIGURELLI, Michele, PANTANO, Linda, SGRÒ, Enza, op. cit., p. 21.

39 AGS, fondo Salvo Riela (d'ora in poi SR), b. Giuseppe Maniaci (corsivo mio).
} 
conti effetto dei suoi trascorsi criminali. Vedremo come quest'idea di punizione meritata si sia consolidata fra i carabinieri che seguirono il caso. L’ipotesi della vendetta personale oscura ed esclude già nei primi passi delle indagini la pista politica, che viene però sottolineata dai compagni della vittima. Il successivo rapporto dei carabinieri del 4 novembre dello stesso anno, ribadisce ancora di più l'estraneità politica nell'uccisione sostenendo che è:

esclusa la peregrina idea del delitto politico, messa fuori in malafede dai suoi compagni di partito [...] a Terrasini la politica non trova terreno fertile ${ }^{40}$.

L'idea del movente politico oltre a non essere neanche presa in considerazione come possibile, viene considerata come una strumentalizzazione da parte del partito a cui era iscritta la vittima. Il Maniaci nel primo verbale viene descritto come un ladro, il che spiegherebbe il movente di una vendetta da parte di coloro che avevano subìto la sua azione criminale. L'unico reato accertato che Giuseppe Maniaci commise fu quando, tre mesi prima della sua uccisione, venne fermato con un sacco di carrube rubato. Nel verbale del processo redatto dal maresciallo Luigi Licata viene sviluppata una criminalizzazione della vittima, Maniaci viene descritto come un pericoloso delinquente e non come un ladro di campagna che avrebbe potuto infastidire qualcuno. Sicuramente Maniaci doveva aver infastidito qualcuno se nel verbale redatto viene menzionata la mafia.

Quali i moventi del delitto? Solo vendetta personale, quale regolarizzazione di conti, perché i mafiosi del luogo non sopportavano questi furti41.

L'idea di una mafia che punisce i ladruncoli di campagna sembrerebbe essere un'ammissione del suo ruolo di garante dell'ordine da parte dei carabinieri. La figura del Maniaci assume aspetti sempre più criminali nella stesura del verbale che mette in luce un approfittatore e parassita dedito al «furto in cui il Maniaci si era da tempo abituato e per cui incuteva soggezione, specie nella parte dei piccoli borgesi [sic], i quali mal sopportavano che il Maniaci, senza stenti e sudori, menasse vita piuttosto comoda, a spese del loro lavoro [...]»42. Ma ciò che rende il caso di Giuseppe Maniaci particolare ed unico sta nell'affermazione seguente, riportata nel verbale:

40 Rapporto dei carabinieri del 4 novembre 1947 riportato in FIGURELLI, Michele, PANTANO, Linda, SGRÒ, Enza, op. cit.,p. 32 (corsivo dell'autore).

${ }^{41}$ AGS, fondo SR, b. Giuseppe Maniaci (corsivo dell'autore).

42 Ibidem. 
[...] Ma se il piccolo agricoltore per timore del peggio taceva e sopportava, lo stesso non doveva verificarsi con i limitrofi Di Maggio Procopio, Vitale Leonardo, Di Maggio Giuseppe, tutti da Cinisi ben noti per la loro appartenenza alla maffia [sic] e soprattutto conosciuti come gente da non subire angherìe43.

La mafia non solo viene nominata ma vengono fatti addirittura i nomi di personaggi in qualità di appartenenti alla "maffia" locale, visti quasi come dei vendicatori dei torti subiti. È indubbio che da questa affermazione trapeli un atteggiamento di favorevole disposizione verso la mafia, vista come una protettrice del «piccolo agricoltore che per timore del peggio taceva». La conclusione del verbale rende evidente la criminalizzazione del Maniaci: la sua uccisione è stata causata dal suo comportamento criminale più che dalla mafia che ha soppresso un dirigente della Federterra locale.

[...] Da quanto precede e dall'insieme delle circostanze emerse nel corso delle indagini, si è addivenuti alla conclusione, comunemente formata nell'ambiente locale, che il Maniaci Giuseppe sia caduto vittima della sua cattiva condotta e che gli uccisori siano gente appartenente alla nota maffia di Cinisi, la quale si è ormai specializzata nel liquidare tutti coloro che danno fastidio alle persone e alla proprietà44.

Non poteva essere più netta un'affermazione di ammissione di convivenza e connivenza con il potere mafioso da parte delle stesse forze di pubblica sicurezza. Viene vista sotto una luce positiva una mafia a difesa dell'ordine pubblico e della proprietà privata contro i turbolenti movimenti sociali del dopoguerra guidati dai dirigenti del movimento contadino, che con le loro azioni e con le loro politiche destabilizzavano la condizione di equilibrio e di accordo raggiunta tra agrari, politici e mafiosi. D’altronde la richiesta dell'applicazione del decreto Gullo n.279 non poteva che suscitare una forte repressione da parte dei custodi dell'ordine tradizionale 45 . Curiosamente il rapporto dei carabinieri giungeva alla conclusione che l'omicidio del segretario della Federterra di Terrasini fosse opera di ignoti, non considerando i nomi dei mafiosi precedentemente fatti. Il Giudice istruttore chiese spiegazioni di questi precisi riferimenti al maresciallo che aveva compilato il verbale citando i nomi di mafiosi del luogo, nonché vicini di

\footnotetext{
43 Ibidem.

44 Ibidem (corsivo e grassetto dell'autore).

45 Il conflitto che il decreto fece nascere dappertutto nelle campagne fu tale che venne definito «una legge dello Stato contro lo Stato degli organi dirigenti nazionali» in un articolo apparso su «La Voce di Sicilia» del 25 ottobre 1945.
} 
Maniaci. Si evince dai verbali un sistema di relazioni interne al paese che permetteva di far dichiarare al maresciallo - senza per questo avere delle prove giudiziarie -i nomi di quelli riconosciuti dall'“ambiente locale" come mafiosi. La dichiarazione di risposta del maresciallo non consentì di indirizzare le indagini verso quei sospetti per mancanza di prove: «abbiamo formulato per contro nostro sospetti [...] ma nulla abbiamo potuto acclarare a loro carico, non essendo neanche risultato che avevano denunziato di avere patito dei furti»46. Nonostante l'individuazione dei mafiosi che avrebbero potuto essere gli autori del delitto le indagini furono sospese e poi concluse con una declaratoria di improcedibilità perché ignoti gli autori del delitto. La responsabilità del delitto venne attribuita direttamente alla stessa vittima e al suo comportamento: la sua condotta sarebbe stata la vera causa della morte.

\subsection{Placido Rizzotto}

Il rifiuto ad indagare sul movente politico delle uccisioni verificatesi nella Sicilia del dopoguerra andava contro le proteste dei compagni delle vittime. Nel caso di Maniaci si è potuto vedere come il movente di natura politica venne subito escluso, ridotto ad una «peregrina idea» usata per strumentalizzare l'omicidio di un poco di buono e «messa fuori in malafede dai suoi compagni di partito». Anche per l'omicidio del sindacalista Placido Rizzotto, avvenuto a Corleone il 10 marzo del 1948, si verificò una simile volontà di insabbiamento del movente politico, anche se in questo caso i carabinieri lo dovettero includere tra i possibili moventi a causa della forte mobilitazione, non solo dei compagni di Rizzotto, ma anche dell'opinione pubblica nazionale. Carabinieri, polizia e magistratura vennero sollecitate nelle indagini dall'interesse nazionale che ebbe tale omicidio ma «rimasero esitanti sulla pista da battere e sulle motivazioni del delitto. Non è chiaro se ciò avvenne per insufficiente comprensione dell'ambiente e degli avvenimenti, oppure perché ricevettero indicazioni o veti cui si uniformarono» 47 . Nel verbale di denunzia fatto il 3 aprile, il comandante della Compagnia dei carabinieri di Corleone, il capitano Filippo Rosati - insieme con il comandante della locale sezione dei carabinieri, Generoso Tozzo - scrisse che «il movente [...] è l'attività di organizzatore sindacale di Rizzotto esponente del Movimento contadino diretto alle occupazione delle terre incolte»48. Questa dichiarazione, diretta e inusuale per le forze di sicurezza del periodo, avrebbe condotto le indagini verso chi si opponeva al

\footnotetext{
46 In Ibidem.

47 FIGURELLI, Michele, PANTANO, Linda, SGRÒ, Enza, op. cit., p. 39.

48 Riportato da Ibidem.
} 
movimento e aveva quindi interesse nell'uccisione di Rizzotto, ma fu parzialmente smentita dai successivi verbali. Nella dichiarazione del 20 maggio rilasciata dal capitano Tozzo (divenuto comandante della Compagnia dei carabinieri di Corleone al posto di Rosati) al giudice istruttore è riscontrabile oltre al cambio di cariche e un mutamento di opinione: «secondo me, anche i familiari del Rizzotto [...] hanno voluto prospettare il delitto come determinato da movente politico per avere, come hanno ottenuto dal Partito aiuto e soccorsi finanziari»49. Il nuovo comandante della stazione dei carabinieri, il Maresciallo Maggiore Nicolò Accomando non dava per certo il movente politico ricercando eventuali motivazioni non solo nel passato della vittima ma anche in quello dei suoi parenti. Dichiarò infatti al giudice istruttore il giorno dopo: «[...] visti i precedenti di Rizzotto Carmelo [...] non è da escludere che la scomparsa del figlio (Placido) sia dovuta a qualche vecchio conto che si è voluto regolare» ${ }^{\circ}$. Le forze dell'ordine indagarono su fatti meno importanti, trascurando il contesto generale in cui si svolse l'omicidio di Rizzotto e il significato che poteva assumere l'uccisione di un sindacalista socialista, dirigente della Federterra e segretario della Camera del lavoro locale, che aveva guidato l'occupazione delle terre incolte a favore delle cooperative contadine. Molti compagni della vittima fecero notare l’importanza che Rizzotto aveva all'interno del movimento e quali minacce ebbe a subire soprattutto nei mesi vicini alle elezioni politiche. La lotta sindacale da lui intrapresa garantì alla cooperativa "Bernardino Verro" ${ }^{1}$ di Corleone la gestione di un intero feudo; le cooperative agricole divennero dei luoghi sociali di opposizione alla classe agrario-mafiosa ${ }^{2}$ e scalzarono il dominio dei gabelloti sui latifondi. Queste lotte e rivendicazioni erano delle sfide dirette al potere mafioso tenuto a Corleone da Michele Navarra e dal boss emergente Luciano Liggio, autore materiale del delitto. L'eliminazione di Rizzotto non fu solo la soppressione di un dirigente del movimento contadino che risultava molto pericoloso per il blocco agrario-mafioso, ma fu anche un diretto messaggio politico. Stando alle dichiarazioni rese da Antonino Di Palermo al giudice istruttore il 2 luglio 1950, dopo la morte di Rizzotto lui e molti iscritti al PSI di Corleone, passarono al Partito Socialista

\footnotetext{
49 Riportato da Ibidem.

50 Riportato da Ibidem, p. 40.

${ }^{51}$ Era dedicata al primo sindaco socialista di Corleone. Dirigente contadino, nel 1892 durante il periodo dei Fasci Siciliani fondò il Fascio di Corleone. Bernardino Verro nel 1914 venne eletto sindaco e nel 1915 venne ucciso dalla mafia.

${ }^{2}$ Scrive La Torre nella relazione di minoranza della Commissione antimafia del 1976: «Non vi è dubbio che il movimento contadino siciliano con la sua parola d'ordine "fuori il gabellotto dai feudi" abbia dato il via ad uno scontro frontale con la mafia [...]». LA TORRE, Pio, Relazione di minoranza, URL:

< http://archiviopiolatorre.camera.it/img-repo/DOCUMENTAZIONE/Antimafia/o3_rel.pdf > [consultato il 17 novembre 2012].
} 
dei Lavoratori Italiani53 nato dalla scissione di Palazzo Barberini voluta da Saragat l'11 gennaio per uscire dall'alleanza social-comunista del Fronte Democratico Popolare (detto anche il Blocco del Popolo, che riuniva PCI e PSI54). Vi erano motivazioni di carattere politico-mafioso che spingevano i socialisti a rompere il patto con i comunisti o a far passare il più possibile i contadini dal PSI al PSLI indebolendo elettoralmente il Blocco del Popolo e, conseguentemente, il movimento contadino, che da una rottura o indebolimento dell'alleanza social-comunista ne sarebbe uscito provato. La ragione politica dell'uccisione dei socialisti durante la campagna elettorale del 1948 viene spiegata lucidamente da Pio La Torre nel 1976, nella sua relazione di minoranza alla Commissione antimafia. Le sue considerazioni in merito al rapporto tra uccisioni mafiose e politica nazionale meritano di essere riportate:

Nel corso di quella campagna elettorale [1948] furono compiuti alcuni dei più efferati delitti di mafia contro esponenti del movimento contadino siciliano. Vogliamo ricordare in modo particolare tre episodi: Placido Rizzotto a Corleone, Epifanio Li Puma a Petralia, Cangelosi a Camporeale, dirigenti contadini di queste tre zone fondamentali nella provincia di Palermo e socialisti. Perché tre socialisti? Gli assassinii si susseguirono a distanza di pochi giorni. Vi era stata la scissione socialdemocratica e il movimento contadino in Sicilia restava, invece, unito; occorreva, dunque, dare un colpo al movimento e da parte della mafia si sviluppò una campagna di intimidazioni verso $i$ dirigenti socialisti55.

Liggio e gli altri due sicari mafiosi vennero assolti per insufficienza di prove. Il mandante, Michele Navarra, fu mandato al confino di polizia dopo che si riconobbe la sua pericolosità sociale grazie anche all'azione del giovane capitano Carlo Alberto Dalla Chiesa $^{56}$. Ma il confino durò ben poco: in capo a qualche mese Navarra tornò a Corleone perché il provvedimento venne revocato; non appena rientrato lasciò il partito liberale per avvicinarsi a quello democristiano57. Quelle indagini si risolsero quindi con assoluzioni per insufficienza di prove e nessun colpevole venne condannato per l'omicidio di Rizzotto, del cui cadavere vennero trovati solo dei resti in una foiba nel

\footnotetext{
53 Successivamente cambiò nome in Partito Socialista Democratico Italiano.

54 Dopo la scissione di Palazzo Barberini il Partito Socialista Italiano di Unità Proletaria di Nenni cambierà nome in quello di Partito Socialista Italiano.

55 Ibidem (corsivo dell'autore).

${ }^{56}$ Quest'ultimo scrisse in una nota al giudice istruttore il 30 maggio 1950 che don Navarra «[...] è notoriamente, da alcuni anni, l'esponente di quella mafia corleonese cui vorrebbesi fare risalire la soppressione del sindacalista Rizzotto [...]» (corsivo dell'autore) riportato in FIGURELLI, Michele, PANTANO, Linda, SGRÒ, Enza, op. cit., p. 42.

57 Riportato in Ibidem.
} 
2009. Solamente il 9 marzo 2012 si ebbe la conferma che quei resti fossero effettivamente del sindacalista ucciso dalla mafia; il 24 dello stesso mese gli furono dedicati i funerali di Stato. L'uccisione di Rizzotto - insieme a quelle, nel 1948, degli altri dirigenti sindacali - erano dovute ad un disegno mafioso di arginamento e repressione del movimento contadino che con le occupazioni delle terre incolte metteva a rischio il potere mafioso. La repressione riguardò principalmente i partiti di sinistra aderenti al Blocco del Popolo in quanto maggiormente coinvolti nelle rivendicazioni del movimento contadino. Ma l'intreccio mafia-agrari e politica aveva iniziato a dare prova della sua crudeltà ben prima della campagna elettorale per le elezioni del 1948 e delle uccisioni mirate che servivano a scompaginare il movimento contadino e il partito socialista. La repressione agrario-mafiosa aveva già dato il suo massimo esempio di violenza anticontadina in quella che per molti versi è da considerarsi come la prima strage di Stato dell'Italia Repubblicana: la strage di Portella della Ginestra58.

${ }^{8}$ Questa è la tesi sostenuta da CASARRUBEA, Giuseppe, Storia segreta della Sicilia, cit. 


\section{* L'autore}

Gabriele Montalbano, è studente del Master in "Histoire des sociétés occidentales contemporaines” all'Università di Parigi 1 Panthéon-Sorbonne e all’École Normale Supérieure di Parigi, dove sta svolgendo ricerche sulla storia politica e culturale del Mediterraneo sotto la tutela di Gilles Pécout e di Pascal Ory. Allievo del Collegio Superiore dell'Alma Mater Studiorum - Università di Bologna, ha conseguito la laurea triennale nel 2012 con una tesi in Storia dell'Italia contemporanea dal titolo Le lotte contadine e la cooperazione agricola in Sicilia nel secondo dopoguerra, relatrice la professoressa Marica Tolomelli. Oltre alla storia politica e sociale i suoi interessi riguardano la storia delle migrazioni e delle relazioni culturali transnazionali in età moderna e contemporanea con particolare riferimento allo spazio euromediterraneo.

URL: < http://studistorici.com/progett/autori/\#Montalbano >

\section{Per citare questo articolo:}

MONTALBANO, Gabriele, «La repressione del movimento contadino in Sicilia (1944-1950)», Diacronie. Studi di Storia Contemporanea: Sulle tracce delle idee, 29/12/2012,

URL:< http://www.studistorici.com/2012/12/29/montalbano_numero_12/ >

Diacronie Studi di Storia Contemporanea $\beta$ www.diacronie.it

Risorsa digitale indipendente a carattere storiografico. Uscita trimestrale. redazione.diacronie@hotmail.it

Comitato di redazione: Marco Abram - Jacopo Bassi - Luca Bufarale - Gianluca Canè - Alessandro Cattunar - Alice De Rensis - Barbara Galimberti - Deborah Paci - Fausto Pietrancosta - Matteo Tomasoni - Luca Zuccolo

Diritti: gli articoli di Diacronie. Studi di Storia Contemporanea sono pubblicati sotto licenza Creative Commons 2.5 . Possono essere riprodotti a patto di non modificarne i contenuti e di non usarli per fini commerciali. La citazione di estratti è comunque sempre autorizzata, nei limiti previsti dalla legge. 